\title{
Community Membership Management for Transient Social Networks
}

\author{
Lateef Yusuf, Umakishore Ramachandran \\ School of Computer Science \\ Georgia Institute of Technology \\ Atlanta, Georgia, USA \\ \{imlateef, rama\}@cc.gatech.edu
}

\begin{abstract}
Confluence of technologies represented by geolocation, geo-sensing, context and activity recognition, and smart phones with rich sensors is opening up new avenues for media-rich social interactions for a spectrum of applications from entertainment, to commerce, to emergency response. This paper addresses the challenges in membership management of a transient social network (TSN), a community of users with mobile devices engaging in social activities of common interest within specific temporal and geographical locality (e.g., flea market, emergency response, local auctions, etc.). Creating and maintaining viable transient social communities requires solving a number of significant challenges including managing the dynamically created social graph, maintaining connectivity across heterogeneous nodes and interfaces and efficient message delivery among the nodes in a community.

Micrograph is a middleware for managing community membership in TSNs. It helps nodes to discover and participate with other nodes based on device-level, or application-level attributes. It allows a node to simultaneously participate in multiple distinct social communities by overlaying multiple TSNs on top of the available nodes in the physical network. Micrograph gives complete isolation for the activities of a node in each of the TSNs that a node may be participating in simultaneously and it also gives complete transparency to a participant as to the membership of a TSN in which he/she is involved in. In this paper, we present the design and implementation of Micrograph, a proof-of-concept implementation of the middleware using Android platforms, four applications to show the feasibility of Micrograph, and simulation-based evaluation of the implemented prototype.
\end{abstract}

Keywords: mobile platform, location-based social network, group management, opportunistic computing

\section{INTRODUCTION}

The transient interactions between users in public places carrying mobile phones equipped with integrated technologies such as WWAN, Wi-Fi, Bluetooth, Camera and GPS provide opportunities for collaboration across a range of diverse interests. During these interactions, users can become members of dynamically created communities with other users who share similar interests and are within the same spatial and temporal locality. In this paper, we present a system architecture for enabling the formation of such transient social networks (TSN).

We define a TSN as having the following four attributes: spatial locality, temporal locality, encounter-based community formation, and interest alignment. The first three attributes, namely, the participants being in the same geographical locality, being aligned in time for engaging in activities of mutual interest, and not having to know one another a priori, sets apart a TSN from a traditional social network such as Facebook. TSNs offer the opportunity for users to leverage both the physical and virtual world to opportunistically achieve a common purpose. The participants (via their mobile devices) can form a peer-topeer (P2P) overlay network for exchanging information and activities of mutual interest in a decentralized fashion. Members of the network are able to specify their degree and level of participation according to their spatial and temporal locality, their parameters of interests and the resource availability on their mobile devices.

TSNs also differ in several ways from social networks that offer location-based services to individual users. First, while traditional geo-social networks (e.g., Foursquare and Facebook Places) provide users with the ability to connect with friends, acquaintances and neighbors, they do not support the on-demand formation and membership management of communities of people who may or may not know each other and are willing to collaborate together for a limited time. Second, the requirements for pre-registration, pre-authentication and pre-initialization by existing traditional geo-social networks excludes a large number of encounter-based communities whose members may not have pre-existing associations with a particular social networking service. Furthermore, traditional social networks typically impose business policies that restrict user's ownership of content, the ability to control their degree of participation in the network, and their ability to leave the network at will. Finally, TSNs have the potential to support latency-sensitive communications and to reduce the load on the backbone network infrastructure by exploiting near-neighbor wireless communications (where possible and sufficient) between user devices.

Figure 1 shows a couple of examples of applications exploiting TSNs. The first is Market Street, where sellers in a flea market or a shopping mall can display and advertise their goods to patrons (see Figure 1-(a)). The application allows patrons to view active hot deals and instant reviews of items from other patrons. Ubiquitous Concession Stand (see Figure 1-(b)) is another application that allows customers in a golf course or a football stadium to place orders and receive food and drinks from one of the roving vendors. Car Auction (see Figure 1-(c)) is an application that allows the customers at a car auction to place bids on vehicles, view the history of the vehicles, and compare the vehicles to similar 

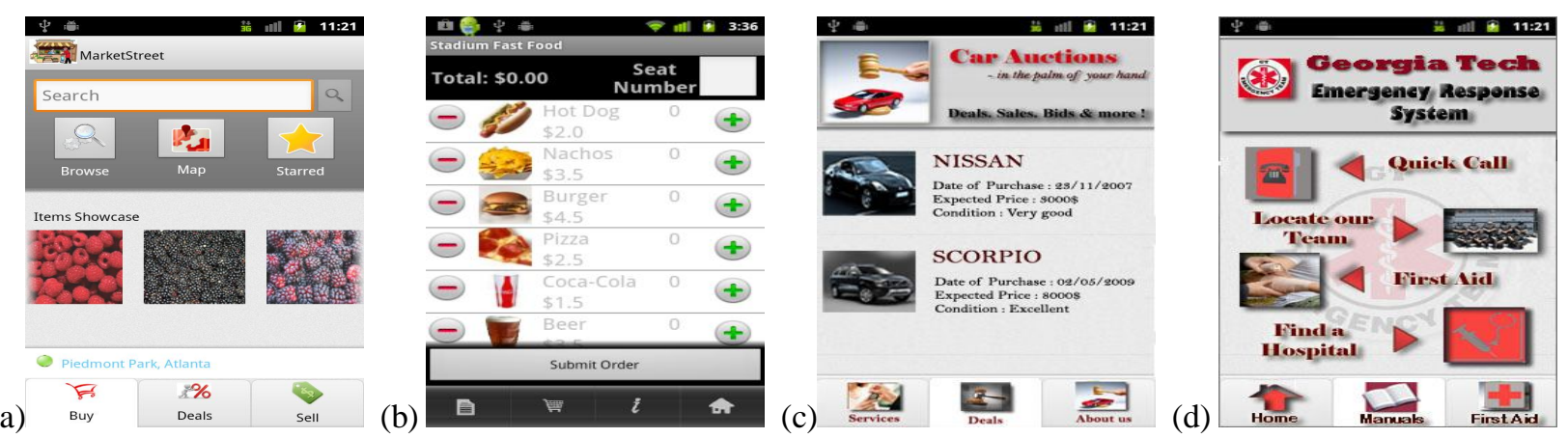

Figure 1. Examples of transient social networking applications: (a) market street; (b) ubiquitous concession stand; (c) car auction; (d) neighborhood emergency response team

vehicles. The application helps customers to have better understanding of the vehicles they are interested in before placing a bid. Neighborhood Emergency Response Team (see Figure 1-(d)) is an application that allows volunteers to collaborate with designated safety officers during large events such as street carnivals, concerts and political campaigns.

To realize the vision of a TSN and enable a variety of applications to be rapidly deployed on a TSN, we need to address a number of significant systems challenges. Since the mobile nodes move in and out of each other's network visibility, realization of a TSN requires the following functionalities to be handled in an autonomic fashion: (a) exporting to the application the membership of the dynamically created social graph, (b) admission of new nodes into existing graphs, (c) merging of graphs that share the same attributes into one, and (d) removing inactive nodes from the graph. Further, the supporting infrastructure for a TSN should implement techniques for handling the diversity and heterogeneity of the nodes in the network and implement efficient application-level messaging between the members of the network.

Micrograph is a middleware for supporting the management of community membership in TSNs. It provides services such as membership management, messaging, and state management in a uniform manner for applications built on top of TSNs. It gives intuitive control to an application for specifying device-level attributes (such as sensing requirements, energy considerations, and network bandwidth), as well as application-level attributes (such as interest in specific types of information and locality) in forming a TSN. Micrograph uses these attributes autonomically in forming and morphing the TSN (e.g., splitting and merging the social graphs when such attributes are violated or met, respectively). In doing this, it ensures that the member list of a community is consistent across the nodes in the community, i.e., it gives complete transparency to the participants as to the instantaneous membership of the TSN graph that a participant is currently participating in. Finally, a participant (i.e., the device associated with her) may be associated with multiple TSNs simultaneously. Micrograph ensures complete isolation for each application that a given device may simultaneously be participating in at any given moment. In a nutshell, Micrograph provides novel mechanisms for community management to address the specific challenges faced by applications that live on top of TSNs.

We have implemented Micrograph on Android platforms configured to support wireless local area networking. As proof of concept, we have constructed four demonstrative applications shown in Figure 1 on top of Micrograph ${ }^{1}$. We have conducted scalability studies of the Micrograph-based approach for a large communities using simulation.

This paper makes the following contributions:

- Design guidelines and system architecture of Micrograph, a middleware for enabling the formation of TSNs.

- A hierarchical organization of the nodes that abstracts network heterogeneity and the vagaries of overlay membership.

- Novel protocols for autonomic membership management of TSNs commensurate with user policies for isolation and resource management.

- A proof-of-concept implementation of Micrograph on Android platforms, demonstrative applications built on top of TSNs.

- Simulation-based evaluation of Micrograph to demonstrate the scalability of the implementation.

The rest of this paper is organized as follows. We position the research presented in this paper in relation to related work in Section II. We present the system architecture in Section III and the organization of the social graph in Section IV. The experimental setup and evaluation results are covered in Section V. We present concluding remarks in Section VI.

\section{RELATED WORK}

The work presented in this paper builds on considerable prior research on topics including ad hoc networking, location-based social networking, group formation, and urban sensing.

Location-based social networking has become recently popular with systems such as Facebook Places [2], Foursquare [3], and Socialight [4]. These systems focus on

Undergraduates mentored by Lateef Yusuf won research awards at the 2011 Annual Research Symposium for demonstrating applications on top of Micrograph [1]. 
the concept of "friending" or building relationships between existing friends or potential friends with similar interests. They also associate (non-temporal) relationships between participants and specific geo-locations. These systems differ from Micrograph in that they do not provide a convenient platform for organizing groups of disparate local users into distinct communities. Micrograph offers several qualitative advantages over these centralized systems including data connectivity costs savings, on-demand formation and membership, scalability, and support for latency-sensitive communications.

Micrograph also shares similarities with a number of participatory and community sensing systems $[5,6]$ such as Micro-blogs [7], and PRISM [8]. In Micro-blogs, users upload "blogs" annotated with sensed information such as photos to a server. The blogs are tagged by location coordinates that the users upload to a server periodically. The platform can then process location-based queries by other users. Similarly, Mob [9] and PeopleNet [10] provide distributed querying to mobile devices by mapping queries to geographic locations called Bazaars. Both Micrograph and these platforms involve a group of nodes collaboratively involved in certain sensing or querying tasks on smartphones. In contrast to these systems, Micrograph is designed for continuous connectivity and exchange of information between nodes rather than a per-instance querying platform.

A number of opportunistic and ad hoc networking platforms such as Spontaneous networking [11], Haggle [12], and MobiClique [13] use a pub/sub interface for content dissemination among mobile nodes. These systems differ from Micrograph in that they are data-centric publish/subscribe approaches that focus on interest forwarding. Given that these systems are focused on content dissemination and not community formation, they do not provide hooks for the applications to interface with the networking layer to manage neighbor views, or provide facilities for nodes to specify resource, location, or sensing requirements for construction of transient social communities.

Group formation and management has been studied by prior work in specific contexts such as peer to peer networking, distributed systems, sensor networking, and opportunistic networking [14, 15, 16, 17]. Micrograph introduces group management techniques that take the location, battery availability, registered interests, and other individual node requirements into consideration for maintaining, splitting, and merging groups of nodes in TSNs.

In summary, while previous platforms or proposals share similarities with the Micrograph platform, they solve complementary problems to what we are focused on in this work. Specifically, our focus in Micrograph is to design techniques that effectively address the problem of creating and managing TSN communities by adapting to user and node criteria such as location, interests, sensors, and resource requirements while exploiting device proximity and heterogeneity. We are not aware of any other system that provides the features that we believe are common requirements for a wide range of transient social networking applications.

\section{SYSTEM ARCHITECTURE}

\section{A. Design Principles}

Micrograph has four major design principles: (1) to support a broad range of encounter-based transient social communities, (2) to provide transparency from third parties and centralized servers (i.e., activities within a community are only visible to members of the same community), (3) to provide isolation among the currently running applications on a user device (i.e., an application can only interact with other devices that match its interest sets, sensing requirements and resource availability), and (4) to provide good performance by exploiting wireless networks. These principles help to capture the unique properties and functionalities of TSNs as stated in Section I.

\section{B. System Components}

Figure 2 shows the software module that runs on each mobile device. Each node may have differing degrees of resource availability and network connectivity. For example, in the car auction scenario, the auction house may augment the personal smart phones of the patrons with resource-rich nodes that can form a cloudlet [18] and provide latencysensitive computation support while being connected to cloud servers on the Internet.

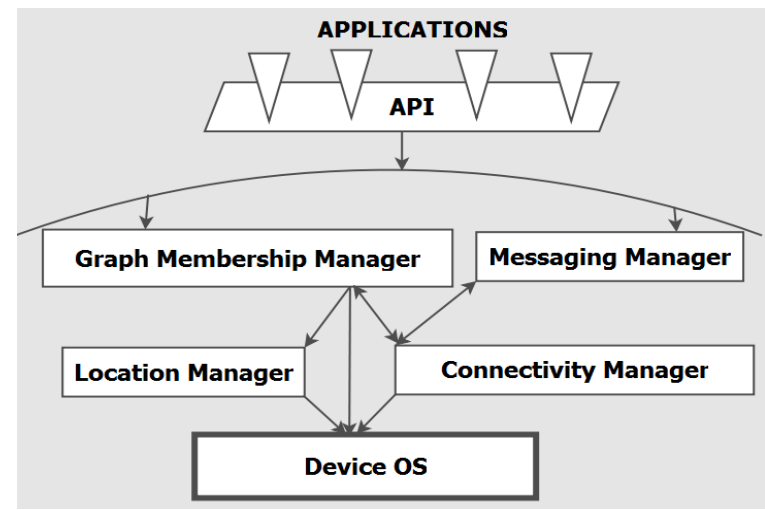

Figure 2. Micrograph architecture

We assume that each device has some facility for determining its current location, detecting changes in movement, measuring resource usage such as battery availability and interacting with sensors present on the device. These artifacts are determined with the help of the native facilities provided by the operating system running on the device (shown as device $O S$ in Figure 2).

The location manager interacts with the device $O S$ to determine the device location using an available localization technology on the device such as GPS localization or wireless network localization.

The graph membership manager running on each device manages community membership functionality for the applications running on the TSN. Depending on an application's interest set, sensing needs, and current locality, 
the graph membership manager informs the application of the dynamic joining and leaving of other participants that meet those requirements.

The messaging manager is responsible for coordinating the exchange of application-specific information between members of an existing graph. It allows an application to send and receive messages to individual members or a subset of its membership list.

The data connection between peers in the TSN is managed by the connectivity manager. Micrograph supports a number of connectivity configurations including: (a) an adhoc Wi-Fi or Bluetooth network, (b) a network of mobile devices connected to a municipal Wi-Fi network, nearby Wi$\mathrm{Fi}$ hotspots or private access points (e.g. a campus or apartment community Wi-Fi network), (c) data connections through the cellular networks, and (d) a hybrid network that opportunistically exploits each of these possible connectivity alternatives. There are several works [19, 20] that have examined how to leverage the combinations of the data connectivity available to a device in an opportunistic manner. Also, in our previous work [21], we outlined techniques for dynamic connectivity management in a TSN. In this paper, we do not discuss the specific details of those connectivity features any further.

TABLE I. MICROGRAPH API

\begin{tabular}{|c|}
\hline Community Definition \\
\hline $\begin{array}{l}\text { interestId = createInterest(name); } \\
\text { commId = createCommunity(coords, duration); } \\
\text { addInterest(commId, interestId); } \\
\text { removeInterest(commId, interestId); } \\
\text { joinCommunity(commId); } \\
\text { listCommunities(); }\end{array}$ \\
\hline $\begin{array}{l}\text { Member Set Manipulation } \\
\end{array}$ \\
\hline $\begin{array}{l}\text { event addMember(memberId); } \\
\text { event removeMember(memberId); } \\
\text { boolean available = isMember(memberId); } \\
\text { location = queryMemberLocation(memberId); } \\
\text { interestSet = queryMemberInterests(memberId); } \\
\text { updateCurrentLocation(location); }\end{array}$ \\
\hline Messaging \\
\hline $\begin{array}{l}\text { boolean sendToMember(msg); } \\
\text { boolean sendToMemberSet }(\mathrm{msg}) \\
\mathrm{msg}=\text { receiveFromMember }() \\
\text { msg = receiveFromMemberSet }()\end{array}$ \\
\hline
\end{tabular}

Table I summarizes the Micrograph API. The API is divided into three categories: community definition, member set manipulation, and messaging. To manage the dynamism in the social graph, Micrograph allows applications to register for specific events (addMember and removeMember methods) when a member node that matches its community requirements joins or leaves the network; as well as primitives for querying information about other member nodes such as location and interest set. We do not claim that the API is complete or is exhaustive; further enhancements may be needed to fully support future evolutions of applications built for TSNs. The API as specified in Table I is versatile enough for the focus of this paper, namely, community membership management.
In the next section, we describe the details of the community membership management, which is the focus of this paper.

\section{ORGANIZATION OF THE SOCIAL GRAPH}

\section{A. Assumptions}

For the nodes participating in a TSN, we make a number of intuitive assumptions. First, we assume random mobility of the nodes. We also assume that each node can discover other nodes connected to the same physical network and can send data to and receive data from those nodes using mechanisms available natively on the node. These mechanisms would be implemented differently depending on whether the physical network is a wireless local area network or a cellular network. For example, on wireless local area networks techniques that would be appropriate to use include those presented by Chandra, et al. [22], or Vasudevan, et al. [23] for nodes to discover one-hop or multi-hop neighbors connected via ad hoc routing or connected to neighboring access points. On cellular networks, a TSN would leverage geographically-aware peer-to-peer overlays such as Globase.KOM [24] and GeoPeer [25] for maintaining content distribution between peer nodes. Messages transmitted by each node can then be bounded by limiting them to nodes within the participant's current geographical locality.

While Micrograph can be adapted to meet the specifics and exploit the features of the underlying physical network, in the rest of this paper, we focus on Micrograph design for a TSN deployed on a wireless local area network.

\section{B. Overview of the Social Graph Model}

We hierarchically model the social graph as consisting of three levels: data connectivity network (DCN), feasible overlay graph (FOG), and the transient social network (TSN) (Figure 3). As the name suggests, the DCN includes all the nodes that are capable of communicating with one another via the wireless network (either directly or through intermediary nodes that serve as relays). Further, DCN captures the temporal locality that we alluded to in Section I, namely, all the nodes in DCN are available at the same time for participating in the formation of a TSN. The FOG on the other hand, represents the set of nodes that can feasibly be part of a TSN given application-independent constraints such as relative mobility and battery life. Thus, the nodes in the FOG are a subset of the nodes in the DCN (e.g., $\mathrm{H}$ is in DCN but not in FOG in Figure 3). Lastly, the TSN, as we mention in Section I, is the application's view of the network for the social interactions among its participants, based on interests expressed by a participant. Micrograph facilitates the formation of the FOG using topology management protocols among the members of the DCN, thereby abstracting the heterogeneity of the physical network. Micrograph manages the continuous arrival and departure of new nodes into the FOG. Further, it manages the application's view by overlaying user-specified constraints (e.g., interest set, privacy, etc.) to form a TSN on top of the FOG commensurate with these constraints. 


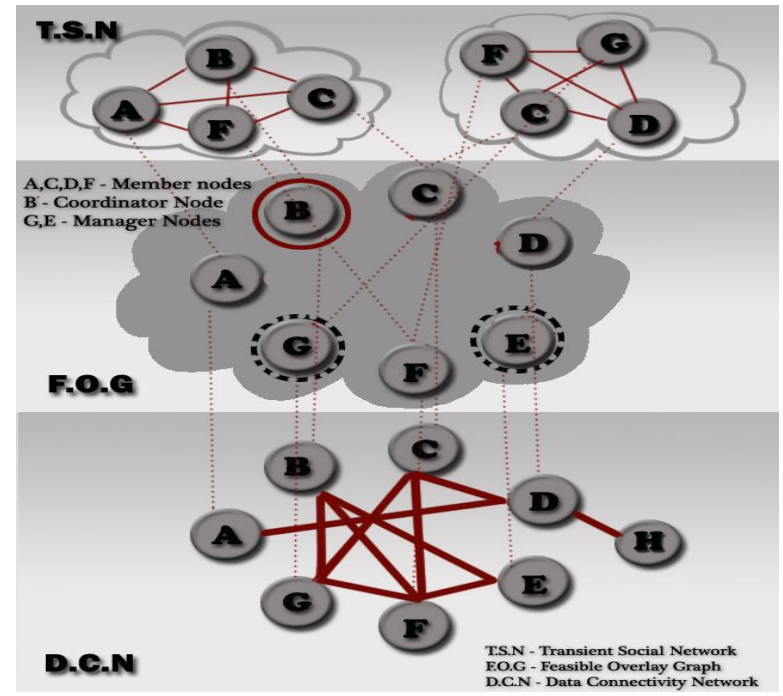

Figure 3. Micrograph social graph model consisting of three levels: Data connectivity network (DCN), Feasible overlay graph (FOG), and distinct TSNs representing disparate communitues as overlays on top of the FOG.

In order to build a scalable TSN, a node in an FOG may assume one of three roles: an ordinary member, a coordinator, or a manager. A node that assumes the role of a coordinator (node B in Figure 3 ) is overall responsible for the maintenance and evolution of the associated FOG. It is responsible for enforcing constraints on graph membership, inter-graph communication between disjoint graphs, coordinating the merging of two FOGs whenever necessary, and the transfer of nodes from one FOG to another. As new nodes join the FOG and the graph membership increases, the coordinator node may assign one or more manager nodes $(\mathrm{G}$ and $E$ in Figure 3) to assist in graph management operations. Each manager node is responsible for maintaining the state information for a disjoint subset of the nodes in the FOG,

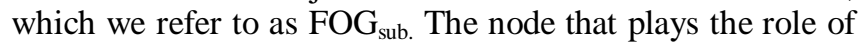
the coordinator is additionally responsible for coordinating the exchange of state information among the manager nodes.

The FOG membership application-independent constraints are meant to ensure effective communication among the nodes in an FOG despite the dynamism in the network due to node mobility, health of a node, etc. Examples of such constraints include:

- Safe Distance: an upper bound on the physical distance between any two nodes.

- Graph Size: maximum membership for an FOG.

- Node Stability: an upper bound on the number of join/leave by a node that is tolerable, which serves as a measure of the node mobility.

Micrograph implements a number of membership management protocols for nodes in a TSN. The goal of Micrograph's membership management protocols is to present to the applications on top of TSN an accurate view of the nodes that are actually available for participating in a social activity given the vagaries of the network and the mobility of the participants. To achieve this goal, Micrograph may configure and reconfigure an FOG in response to events such as (a) the joining of a new node, (b) the departure of an existing node, (c) the selection of a new graph coordinator, and the (d) merging of two FOG instances. Due to space limitations, we do not discuss the details of Micrograph's membership protocols in this paper. The details of the protocols are available in the following technical report [27].

\section{EXPERIMENTAL SETUP AND EVALUATION}

We have implemented the Micrograph runtime in Java encompassing the community membership management protocols described in Section $\mathrm{V}$ as a middleware on top of Android 2.3 OS. As a proof of concept of Micrograph, we have built four applications on top of the Micrograph platform: Market Street, Ubiquitous Concession Stand, Car Auction, and Neighborhood Emergency Response Team. For quantitative evaluation, we use a custom simulator designed for this project to evaluate the scalability and effectiveness of our membership management protocols. There are three experiments: (1) The first one measures the admission delay for a node to join an existing TSN; (2) The second one illustrates the ability of Micrograph to stabilize under node churns and readmissions. (3) The third experiment measures the overhead (in terms of additional network traffic) for the existing nodes when a new node joins the TSN.

\section{A. Simulation Model}

The mobile nodes in our simulations implement the graph formation manager of our architecture (Figure 2). The network area is a 1000 meters $\times 1000$ meters topology, the transmission rate of each node is $54 \mathrm{Mbps}$ and the default radio range is 250 meters. Each node moves within the network area according to the random waypoint mobility model [26]. The velocity of a node is evenly chosen from a uniform distribution of 1 meters/s minimum and 1.8 meters/s maximum, with a uniformly distributed pause time of $60 \mathrm{~s}$. Each member node sends status updates to its manager or coordinator node at a periodic rate of $500 \mathrm{~ms}$. We use a safe distance of 500 meters and a maximum FOG $_{\text {sub }}$ size of 100 for most of the simulation experiments. The simulation environment models a scenario whereby nodes within a geographical area (e.g. campus network) are walking at a moderate pace with occasional stops.

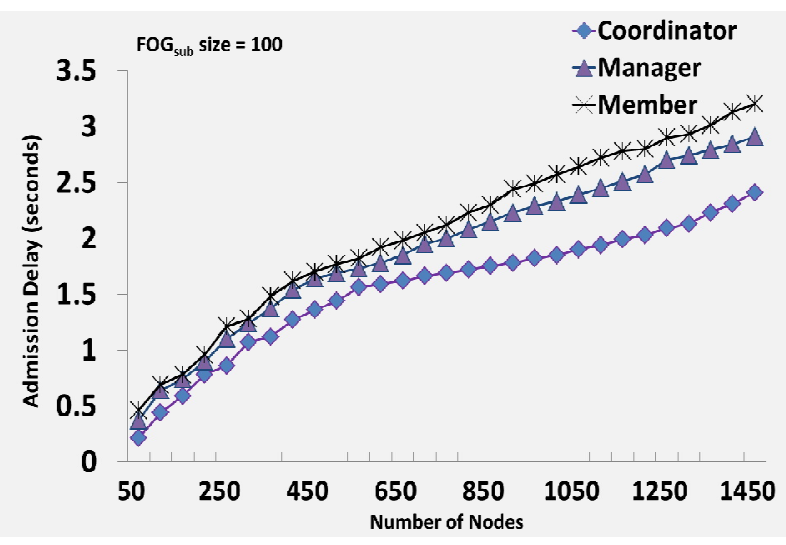

Figure 4. FOG admission delay. 


\section{B. Delay due to Node Admisssion}

We define the admission delay as the time it takes for existing members of the FOG to become aware of a new node joining the FOG. The first experiment measures the admission delay for a new node joining an existing FOG. Using a maximum $\mathrm{FOG}_{\text {sub }}$ size of 100 , we gradually increase the number of nodes in a given FOG from 1 to 1500 . The results are shown in Figure 4. The figure shows the admission delay as a function of the graph size.

The results show that the delay is low for all categories of nodes in an FOG. The coordinator node has the least admission delay compared to manager nodes and member nodes, because it is the first point of contact by a new node wanting to join the FOG.

\section{Stability of Micrograph under Churn and Readmission}

The second experiment evaluates the behavior of the FOG under repeated membership changes (node churns and node readmissions). We divide the duration of the experiment into 200 intervals; each interval is 30 seconds.

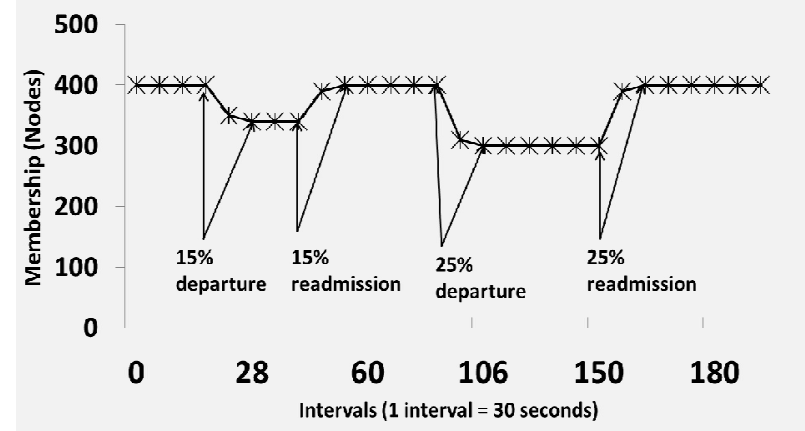

Figure 5. FOG membership under node churns and readmission over 200 intervals.

As shown in Figure 5, during the experiment, we disconnected $15 \%$ of the nodes simultaneously from the FOG between the $22^{\text {nd }}$ interval and the $27^{\text {th }}$ interval and then readmitted them between the $50^{\text {th }}$ and $55^{\text {th }}$ intervals; similarly, we disconnected $25 \%$ of nodes between the $120^{\text {th }}$ and the $125^{\text {th }}$ intervals and readmitted them during the $150^{\text {th }}$ and $155^{\text {th }}$ intervals. We repeated the experiment 100 times. Figure 5 shows how the membership of the FOG evolves during these churns and readmissions. The membership stabilizes within 1 interval subsequent to the churn or readmission, and thus illustrates Micrograph's ability to react quickly to sudden sizable changes in node arrivals or departures from a given FOG.

\section{Bandwidth Overhead due to Node Admission}

We define bandwidth overhead for a node as the additional network traffic incurred by the node during node admission. The bandwidth overhead for a node in an existing FOG is a function of node admissions and node churns. We measure the average bandwidth overhead (both upstream and downstream) experienced by nodes in a given FOG, using a FOG $_{\text {sub }}$ size of 100 .

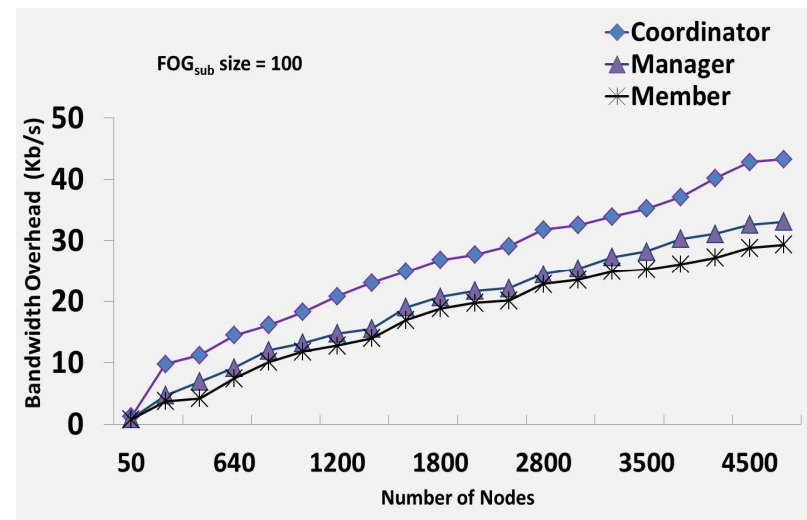

Figure 6. Bandwidth overhead of an FOG as the size of FOG is progressively increased from 1 to 5000 .

The results in Figure 6 show that the bandwidth overhead is low for the different categories of nodes in a given FOG. The coordinator node has the largest overhead, since it receives membership updates from the manager nodes and admission requests from member nodes while the member nodes has the least overhead since it only communicates with its assigned manager node after becoming an FOG member. It is important to note that the overhead grows linearly with the size of the FOG implying that the implementation is scalable.

The last experiment is measuring the bandwidth overhead at the coordinator node as a function of the $\mathrm{FOG}_{\text {sub }}$ size. Figure 7 shows the bandwidth overhead at the coordinator node as the maximum size of the $\mathrm{FOG}_{\text {sub }}$ is increased from 25 to 1000 nodes. The figure shows approximately linear decrease in the overhead, which is what one would expect to see.

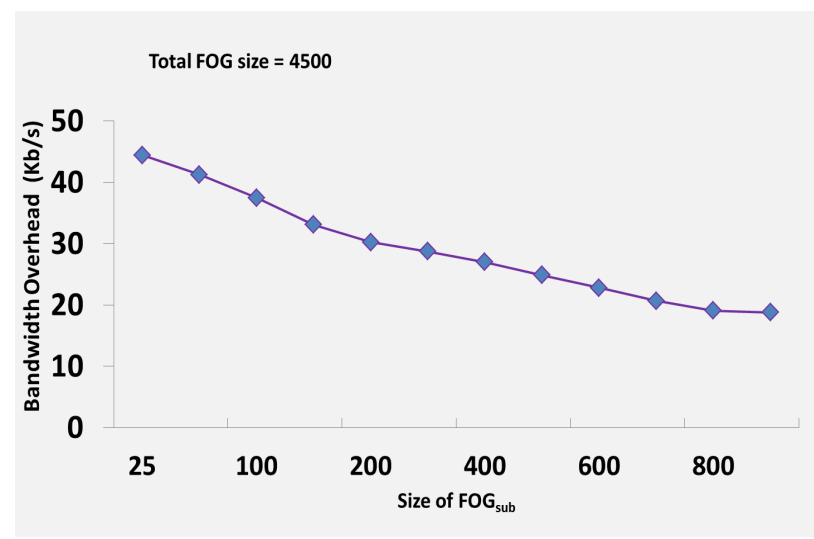

Figure 7. Bandwidth overhead of a coordinator node as the size of FOG $_{\text {sub }}$ is progressively increased from 25 to 1000 .

\section{E. Discussion of Results}

The experimental results confirm the following: (1) Micrograph design ensures that the delay for node admission 
is linear in the number of nodes; (2) The design ensures that it can quickly stabilize following node churns and readmissions; and (3) the bandwidth overhead during node admissions in linear in the number of nodes.

\section{CONCLUSION}

In this paper, we have introduced the concept of transient social networks (TSNs) and presented the challenges in community membership management of TSNs. We proposed novel protocols for meeting these challenges via Micrograph, a middleware that allows autonomic management of community membership for TSNs. We presented the system architecture of Micrograph, which in addition to managing all the dynamism involved in community membership management also manages messaging among the peers of the TSN.

Micrograph offers several qualitative and quantitative advantages for realizing TSNs. It enables social networking activities in environments with inadequate infrastructure support. It helps nodes to discover and participate with other nodes based on device-level, or application-level attributes. Via user-specified attribute filtering, Micrograph allows a node to personalize a given TSN that it is a part of. Micrograph allows a node to simultaneously participate in multiple distinct social communities by overlaying multiple TSNs on top of the available nodes in the physical network. Micrograph gives complete isolation for the activities of a node in each of the TSNs that a node may be participating in simultaneously. It also gives complete transparency to a participant as to the membership of a TSN in which he/she is involved in.

We have implemented Micrograph as a middleware on top of Smartphones running Android OS. As a proof of concept, we have built sample applications on top of the Micrograph middleware. We have quantitatively shown that Micrograph is a scalable platform for maintaining community membership information among nodes in a wireless network.

This paper has addressed the community membership aspect of the TSN research. There are a number of problems that need to be addressed including reliable messaging, privacy-aware protocols, trust-model for participating in the social network, context-aware and resource-aware runtime for programming TSNs, etc. Exploration of such issues is part of our future research.

\section{REFERENCES}

[1] C. Lee, F. Lotfi and J. Wang (2001, Apr.) Micrograph: An infrastructure for spontaneous social networks, UROC Research Symposium, Third Place Judges' Award and Second Place People's Choice Award; Graduate Student Advisor: Lateef Yusuf; Faculty Advisor: Umakishore Ramachandran. [Online] Avalaible: http://www.cc.gatech.edu/research/undergrad/researchsymposium.

[2] (2011) Facebook Places. [Online]. Available: http://www.facebook.com/places/

[3] (2011) Foursquare. [Online]. Available: http://www.foursquare.com/

[4] (2011) Socialight. [Online]. Available: http://www.socialight.com/

[5] A. Krause, E. Horvitz, A. Kansal, and F. Zhao, "Towards community sensing," in IPSN, 2008.
[6] D. Burke et al., "Participatory sensing," in World Sensor Web Workshop, 2006.

[7] S. Gaonkar, J. Li, R.R. Choudhury, L. Cox, and A. Schmidt, "Microblog: sharing and querying content through mobile phones and social participation," in MOBISYS, 2008.

[8] T. Das, P. Mohan, and V. N. Padmanabhan, "PRISM: Platform for remote sensing using smartphones," in MOBISYS, 2010.

[9] R. Chakravorty, S. Agarwal, and S. Banerjee, I. Pratt, "Mob: a mobile bazaar for wide-area wireless," in MOBISYS, 2005.

[10] M. Motani, V. Srinivasan, and P.S. Nuggehalli, "PeopleNet: engineering a wireless virtual social network," in MOBISYS, 2005.

[11] L.M. Feeney, B. Ahlgren, and A. Westerlund, "Spontaneous networking: an application oriented approach to ad hoc networking," in IEEE Communication Magazine, 2001.

[12] J. Su, J. Scott, P. Hui, J. Crowcroft, E. De Lara, C. Diot, A. Goel, M. Lim, and E. Upton, "Haggle: seamless networking for mobile applications," in Lecture Notes in Computer Science, 4717:391, 2007.

[13] A. Pietilainen, E. Oliver, J. LeBrun, G. Varghese, J. Crowcroft, and C. Diot, "MobiClique: middleware for mobile social networking," in WOSN, 2009.

[14] K. Whitehouse, S. Sharp, D.E Culler, and E.A Brewer, "Hood: a neighborhood abstraction for sensor networks," in MOBISYS, 2004.

[15] G.C Roman, Q. Huang, and A. Hazemi, "Consistent group membership in ad hoc networks," in ICSE, May 2001

[16] B. Ford, J. Strauss, C. Lesniewski-Laas, S. Rhea, F. Kaashoek, and R. Morris, "Persistent personal names for globally connected mobile devices," in OSDI, 2006

[17] J. Cowling, D. R. K. Ports, B. Liskov, R. A. Popa, and A. Gaikwad,"Census: Location-aware membership management for large-scale distributed systems," in USENIX '09, 2009.

[18] M. Satyanarayanan, P. Bahl, R. Caceres, and N. Davies, "The case for VM-based cloudlets in mobile computing," in IEEE Pervasive Computing, vol. 8, no. 4, 2009.

[19] A. Balasubramanian, R. Mahajan, and A. Venkataramani, "Augmenting mobile 3G using Wi-Fi: measurement, design,and implementation," in MOBISYS, 2010.

[20] B. Higgins, A. Reda, T. Alperovich, J. Flinn, T. Giuli, B. Noble, and D. Watson, "Intentional networking: opportunistic exploitation of mobile network diversity," in ACM MOBICOM, 2010.

[21] L. Yusuf and U. Ramachandran, "VirtualConnection: opportunistic networking for web on demand," in ICDCN, 2010.

[22] R. Chandra, J. Padhye, and L. Ravindrananth, "Wi-Fi neighborcast: enabling communication among nearby clients," in HotMobile '08, Napa Valley, February 2008

[23] S. Vasudevan, D. Towsley, D. Goeckel, and R. Khalili, "Neighbor discovery in wireless networks and the coupon collector's problem," in MOBICOM, 2009.

[24] A. Kovacevic, N. Liebau, and R. Steinmetz., "Globase.KOM- a P2P overlay for fully retrievable location-based search," in IEEE P2P 2007, pages 87-96, Sep. 2007.

[25] F. Araújo and L. Rodrigues, "GeoPeer: a location-aware peer-to-peer system," in IEEE NCA 2004, 2004.

[26] T. Camp, J. Boleng, and V. Davies, "A survey of mobility models for ad hoc network research," in Wireless Comm. and Mobile Computing (WCMC): Special Issue on Mobile Ad Hoc Networking:Research, Trends, and Applications, vol. 2, no. 5, pp. 483-502, 2002.

[27] Lateef Yusuf and Umakishore Ramachandran,"Micrograph: an infrastructure for transient social networks," Technical Report, School of Computer Science, Georgia Institute of Technology, March 2011 